\title{
Comparison of Two Circuit Class Therapy Programs on Walking Capacity, Gait Velocity and Stair Ambulation among Patients with Chronic Stroke: a Parallel Pretest-Posttest Pilot Study
}

\author{
Kristofferson G. Mendoza, MPT, PTRP, ${ }^{1}$ Maria Eliza R. Aguila, PhD, ${ }^{1}$ Fil Charles S. Alfonso, DPT, PTRP, $, 1,2$ \\ Marianne Grace T. Alfonso, PTRP, ${ }^{1,3}$ Karen D. Elmi, PTRP ${ }^{1}$ and Edward James R. Gorgon, MPhysio ${ }^{1}$ \\ ${ }^{1}$ Department of Physical Therapy, College of Allied Medical Professions, University of the Philippines Manila \\ ${ }^{2}$ Bayhealth Medical Center - Kent Campus, Delaware, USA \\ ${ }^{3}$ Fox Rehabilitation, Delaware, USA
}

\begin{abstract}
Objective. Circuit class therapy is a cost-efficient model of treatment that can be beneficial in a setting with limited resources. Current literature has conflicting results regarding which is a more effective approach to stroke rehabilitation: focusing on functional training or on improving impairments. This pilot study provides preliminary information comparing the effects of a task-oriented versus an impairment-focused circuit class therapy on walking ability among patients with chronic stroke.
\end{abstract}

Method. Eighteen participants with a single episode of chronic stroke and limited mobility were randomized into task-oriented circuit class (task group) ( $n=9$ ) and impairment-focused circuit class (impairment group) ( $\mathrm{n}=9$ ). Both groups underwent intervention thrice a week for four weeks. Blind examination was done using the Ten Meter Walk Test for comfortable gait velocity (CGV) and fast gait velocity (FGV), Time Up and Down Stairs (TUDS), and Six Minute Walk Test (6MWT).

Results. All participants completed the treatment sessions without adverse effects. After four weeks of treatment, the task group showed statistically significant within-group change in CGV $(0.12 \pm 0.08, p=0.003)$ and FGV $(0.25 \pm 0.22$, $p=0.007)$. The impairment group only showed statistically significant improvement in 6MWT $(25.80 \pm 31.2, p=0.038)$. There were no statistically significant changes between the groups in all outcome measures.

Conclusions. The preliminary data from this pilot study suggest either program can improve walking-related outcomes and may not be different, although this needs to be confirmed using an appropriately-powered trial.

Key Words: chronic stroke, circuit-based exercise, task-oriented training, impairment-focused treatment, walking capacity, gait velocity, stair ambulation

\section{INTRODUCTION}

This paper was presented at the Philippine Physical Therapy Association Convention, December 2014, Manila, Philippines and at the World Confederation for Physical Therapy Congress, May 2015, Singapore. The abstract was published in Physiotherapy, Volume 101, pages e995 to e996.

Corresponding author: Kristofferson G. Mendoza, MPT, PTRP Department of Physical Therapy

College of Allied Medical Professions

University of the Philippines Manila

Pedro Gil Street, Ermita, Manila 1000, Philippines

Email: kgmendoza1@up.edu.ph
Stroke is one of the leading causes of disease globally with numerous pervading issues that limit a person's mobility. ${ }^{1}$ In the Philippines alone, the incidence of stroke was estimated to be at a hundred thousand in 2016, causing more than 60,000 deaths and a loss of approximately 1.7 million disability-adjusted life-years (DALYs). ${ }^{2}$ The importance of rehabilitation in improving quality of life after stroke is globally accepted. ${ }^{3}$ In developing countries, however, access to stroke rehabilitation is still limited due to the high incidence of the condition and the shortage of rehabilitation professionals. ${ }^{4}$ There were only an estimated 
13,000 physical therapists in Philippines in 2018 which is disproportionate to the growing number of stroke cases in the country. ${ }^{5}$

Patients who survive a stroke live with the longstanding effects of the condition. One of the main goals of stroke rehabilitation, especially for patients at the chronic stage, is to increase their walking ability, and levels of activity and participation. ${ }^{6}$ Despite improved ambulation with rehabilitation, many patients still have functional impairments even after discharge from the hospital. ${ }^{7}$ Mobility and community participation remain limited for most patients even after six months since the onset of their condition. ${ }^{8}$ Mobility inside and outside the home is further compromised with the decrease in activity of persons with stroke the less active or more sedentary a person with stroke becomes over time. ${ }^{8,9}$

Circuit class therapy is a physical therapy treatment model used in stroke rehabilitation where the patients are treated in groups. Patients move through a circuit of different stations where they repetitively practice functional tasks or exercises in each station. ${ }^{10,11,12}$ The activities are adapted and progressed individually according to the performance of each patient. ${ }^{10}$ Circuit class therapy is cost-efficient because it allows intensive training of several patients with fewer therapists which can be very beneficial in a setting with limited resources. Moreover, because it is conducted as a class, it provides participants with opportunities to interact and learn from each other and provide each other support. ${ }^{11,13}$ Many recent studies on the use of circuit class therapy in stroke rehabilitation have shown its effectiveness in improving standing and walking ability. A meta-analysis by Veerbeek et al found that circuit class composed of mobilityrelated activities improved walking distance, balance, and walking ability regardless of the chronicity of the stroke. ${ }^{14}$ Similarly, a meta-analysis by English et al found that taskoriented circuit class therapy demonstrated superiority over individualized treatment or usual care in improving walking capacity (MD 60.86, 95\% CI 44.55 to $77.17 ; I^{2}=27 \%$ ), gait speed (MD $0.15,95 \%$ CI 0.10 to $0.19 ; I^{2}=14 \%$ ), walking independence (OR 1.91, 95\% CI 1.01 to $3.62 ; I^{2}=34 \%$ ), and ability to stand up, walk, and turn around (MD -3.62, $95 \%$ CI -6.09 to $\left.-1.16 ; I^{2}=0 \%\right) .{ }^{15}$

Task-oriented treatment and treatment focused on improving specific impairments are two conceptually different rehabilitation approaches that are commonly applied in the treatment of chronic stroke. Task-oriented treatment emphasizes repeated performance of specific tasks to address activity limitations. ${ }^{16,17}$ Treatment focused on improving impairments aims to restore faulty body functions such as strength, range of motion, and body symmetry. ${ }^{18} \mathrm{It}$ remains a subject of debate which of the two is the more effective approach to stroke rehabilitation. Current literature on this matter shows conflicting results. There are studies that suggest that the "bottom-up" approach of focusing on impairment is more effective for patients with moderate to severe stroke. ${ }^{19,20}$ Other studies have shown that both approaches are equally effective with no clearly superior approach for improving mobility post stroke. ${ }^{21,22}$ It is still unclear which approach would yield better effects on walking ability.

Most of the studies on circuit class therapy in stroke rehabilitation primarily utilized functional tasks in the treatment protocol. Occasionally, these programs included some exercise stations for strength or fitness. ${ }^{10,11}$ However, majority of the research still consider circuit class therapy to be primarily for task-oriented training. ${ }^{14}$ The effect on poststroke walking ability of a circuit class therapy with stations focused on improving impairments such as strength, weightbearing symmetry, and gait symmetry has yet to be explored. Furthermore, its effects on walking ability have yet to be compared with a more task-oriented circuit program.

The objective of this pilot study was to gather preliminary information to compare the effects of a task-oriented circuit class program and an impairment-focused circuit class program on the walking ability among patients with chronic stroke. Data from this pilot study may provide information to better understand the difference in the effects of the two approaches when applied using a circuit class training model. The results may help inform rehabilitation professionals in selecting better interventions for their patients. Being a pilot study, this can also provide information to determine the feasibility of conducting the same study in a larger scale. Information from this study can help in identifying relevant modifications in research methodology and possible issues in the administration of the two circuit class therapy programs.

\section{MATERIALS AND METHODS}

For this pilot study, we applied a parallel pretestposttest design. ${ }^{23}$ Treatment sessions and data collection were conducted at a university-based outpatient rehabilitation center in Manila, Philippines. Ethics approval was obtained from the Ethics Review Committee of the University of the Philippines Manila College of Allied Medical Professions.

\section{Participants}

This study recruited participants via convenience sampling over a 12 -month period. We used word-of-mouth advertisement to recruit participants from a hospital-based stroke support group and their community-based network. All interested individuals underwent medical screening and were also screened by the authors using interview and assessment of level-ground walking and stair ambulation. They were included if they had a stroke that occurred at least six months ago; and were able to follow three-step instructions in English or Filipino. The authors utilized the criteria by Shumway-Cook et al in determining a person's limited walking ability. At the time of recruitment, volunteers should be unable to walk $0.8 \mathrm{~km}$ of level surface and/or climb 
at least 1 flight of stairs without support or assistance. ${ }^{24}$ However, to be included in the study, they should be able to ambulate at least $10 \mathrm{~m}$ with or without a gait aid to be able to meet the demands of the circuit programs. To prevent other factors from affecting the results of the study, patients already receiving exercise training or physical therapy at the time of recruitment were excluded. Those who had multiple strokes, or unstable or severe cardiovascular, musculoskeletal, and neurological conditions were also excluded from the study. Lastly, those who did not ambulate independently in the community or walked with an assistive device prior to the stroke were also excluded. After screening, eligible participants were oriented to the study protocol and provided written consent. Randomization of the participants to the two groups was done by a person who was not a member of the research team. An equal amount of sealed opaque envelopes for each of the interventions were created. An allocation sequence using a random numbers table was generated and the envelopes were arranged accordingly. For every new participant, the top envelope was chosen to determine the participant's intervention.

Out of the 23 volunteers, eighteen (18) were found eligible for the trial. We excluded five volunteers because they had uncontrolled hypertension, rheumatic heart disease, multiple strokes, or were receiving physical therapy during the time of the trial. Eighteen participants were randomly allocated to either task-oriented circuit class therapy group or the task group $(\mathrm{n}=9)$ or to the circuit class therapy group focused on impairment or the impairment group $(\mathrm{n}=9)$ as shown on Figure 1. Treatment interviews showed that most participants had hemiparesis, asymmetrical gait, limitations in stair ambulation, and limitations in walking outside their homes. The mean onset of stroke was $38.7 \pm 17.5$ months for the task group and $59 \pm 55.4$ months for the impairment group. Overall, demographic characteristics of the two groups were comparable at baseline as shown in Table 1.

\section{Outcome Measures}

A physical therapist with more than 20 years of experience and who was blinded to the group allocation

Table 1. Characteristics and baseline scores of study participants $(\mathrm{N}=18)$

\begin{tabular}{lcc} 
& $\begin{array}{c}\text { Task group } \\
(\mathbf{n = 9 )}\end{array}$ & $\begin{array}{c}\text { Impairment } \\
\text { group (n=9) }\end{array}$ \\
\hline Sex (M/F) & $9 / 0$ & $7 / 2$ \\
Age (years) & $47.2 \pm 8.8$ & $49 \pm 11.2$ \\
Time since stroke (months) & $38.7 \pm 17.5$ & $59 \pm 55.4$ \\
Location of stroke (R/L) & $6 / 3$ & $7 / 2$ \\
Falls in last 3 months prior to & $0.2 \pm 0.4$ & $0.1 \pm 0.3$ \\
the study & & \\
Comorbidities & 8 & 6 \\
$\quad$ Hypertension & 2 & 0 \\
$\quad$ Diabetes Mellitus & 1 & 0 \\
$\quad$ Hyperurecemia & & \\
Barthel Index Score & $92.7 \pm 7.5$ & $91.8 \pm 5.7$ \\
\hline
\end{tabular}

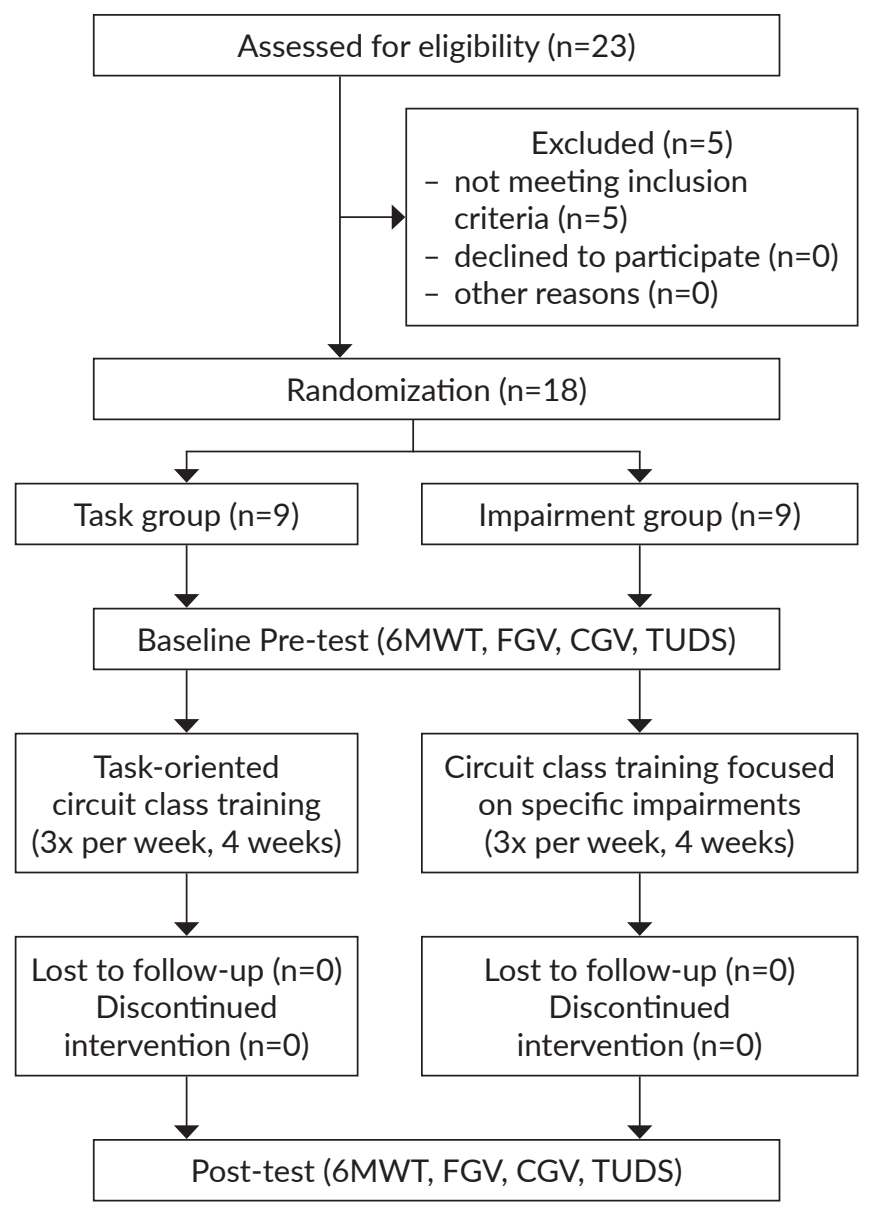

Figure 1. Study flow diagram.

conducted the examination. She assessed the participants within one week prior to the intervention and after completion of the treatment. Four outcomes associated with community ambulation were measured. The Six Minute Walk Test (6MWT) measured walking capacity; the Ten Meter Walk Test (10MWT) measured comfortable gait velocity (CGV) and fast gait velocity (FGV); and the Timed Up and Down Stairs (TUDS) measured the ability to negotiate stairs. These measures had been validated and were shown to have moderate to excellent inter-rater and intra-rater reliability for people with stroke. ${ }^{25-28}$ The minimal detectable change at the $95 \%$ confidence level (MCD95) of CGV and FGV for chronic stroke were $0.18 \mathrm{~m} / \mathrm{s}$ and $0.13 \mathrm{~m} / \mathrm{s}$, respectively. ${ }^{29}$ The minimum detectable change (MDC) among patients with chronic stroke for the $6 \mathrm{MWT}$ was $36.6 \mathrm{~m}$ or a $13 \%$ change while the minimal clinically-meaningful improvement was estimated at 34.4 for chronic stroke. ${ }^{25,26}$

The blinded assessor performed all tests in one session in the same sequence. The 10MWT was tested first followed by the TUDS. The 6MWT were performed last to minimize the effect of fatigue. All outcome measures were administered twice, and the best performance was used for statistical analysis. 


\section{Intervention}

The participants in both groups received the allocated treatment three times a week for four weeks with each session lasting for 60 minutes. The intervention was provided by trained physical therapists with clinical experience ranging from one to ten years, assisted by physical therapy students who were trained in the conduct of the treatment protocol. During the treatment sessions for both groups, one to two therapists were present to oversee the administration of the program. Two to five participants were present during each circuit class therapy session.

In the task group, the participants performed ten different functional tasks as shown in Table 2. These functional tasks mimicked the different mobility demands inside and outside the home. The group was instructed to perform the activities with as many repetitions as possible and as safely as they could for five minutes before moving to the next station. The impairment group performed impairment-focused exercises as shown in Table 3. Exercises were based on the exercise protocol for older adults published by Tufts University and the United States Centers for Disease Control and

Table 2. Task-oriented circuit class therapy

Station Exercise

Station 1 Alternately ascending and descending a ramp and a flight of stairs with at least three steps; the flight of stairs and the ramp hand handrails on one side

Station 2 Walking comfortably for five meters then walking with the fastest speed for another five meters with the distance clearly marked on the floor

Station 3 Heel lifting

Station 4 Standing up from a chair, walking three meters, turning around, walking back, and sitting down on the chair

Station 5 Stepping forward, backward, and sideways onto wooden blocks of various heights

Station 6 Walking on varying surfaces (hard and foam surface) and over obstacles (shoe boxes and cones) set at irregular intervals

Station 7 Walking ten meters while carrying a medium-sized basket to obscure the lower extremities

Station 8 Walking 10 meters while turning head from side to side

Station 9 Standing up and sitting down on chairs of varying height

Station 10 Walking five meters by stepping on floor markers with progressively narrowing base of support

Table 3. Circuit class therapy focused on specific impairments Station Exercise

Station 1 Pelvic bridging held for six seconds

Station 2 Hamstring curls in standing using ankle weights

Station 3 Knee extension in short sitting with ankle weights

Station 4 Hip abduction in standing with ankle weights

Station 5 Forward and lateral step-ups on a four-inch step-up block

Station 6 Lateral, anterior, and posterior weight shifting within parallel bars

Station 7 Walking forwards, backwards, and sideways within parallel bars with turning done for five rounds
Prevention. ${ }^{30}$ The exercises aimed to improve muscle strength, weight-bearing symmetry, and gait symmetry. Exercises for each station lasted for 5 to 7 minutes. The therapists tailored the progression of the exercises to each participant's level of ability. The number of sets were increased when the exercise was deemed no longer challenging enough for the participant.

Rest periods for both circuit class therapy groups were provided, if needed. Both groups started and ended their sessions with supervised standardized warm-up and cooldown periods comprised of walking, and range of motion and stretching exercises of the extremities and trunk for five minutes.

\section{Statistical Analysis}

We reported continuous data such as demographic characteristics as means and standard deviations. When possible, we used the minimum detectable change (MDC) and minimally clinically important change (MDIC) for chronic stroke to interpret the results. In comparing the effects of both interventions on mobility measures, we used the one-way between groups analysis of covariance (ANCOVA), with type of circuit class therapy as the independent variable, best posttest scores as the dependent variable, and pretest scores as the covariate. We also checked the normality, linearity, homogeneity of regression slopes, and equality of variance to ensure that assumptions for the use of ANCOVA were not violated. Alpha level was set at 0.05 . We computed the effect size of the task group in relation to the impairment group using Partial Eta Squared. An effect size of 0.10 is considered small, 0.25 as medium, and 0.40 as large. For the analysis, we used the Statistical Package for Social Sciences statistical software, version 21 (SPSS Inc., Chicago, Illinois, USA) for Windows.

\section{RESULTS}

All participants completed the treatment as allocated (Figure 1). The task group attended an average of $11.66 \pm$ 0.71 sessions while the impairment group $10.78 \pm 1.3$ sessions. Only two absences were allowed to remain eligible to participate in the trial and none of the participants exceeded the limit. The participants did not report any adverse effects in relation to either of the treatment programs.

After 4 weeks of treatment, the task group showed statistically significant within-group change in CGV $(p=$ $0.003)$ and FGV $(p=0.007)$ (Table 4) (Figures $2-5)$. For the impairment group, only the change scores of the 6MWT $(p=0.038)$ showed statistically significant improvements. There were no statistically significant within-group changes for the TUDS scores for both groups. As shown in Table 4 , there were no statistically significant changes between the two groups in all of the outcome measures. All of the outcomes showed small effect sizes. Moreover, only the FGV reached MCD for the task group. The CGV and the 6MWT for both groups did not reach MCD. 
Table 4. Within-group and between-group comparison of the two circuit class programs

\begin{tabular}{|c|c|c|c|c|c|c|c|c|c|c|}
\hline \multirow{3}{*}{$\begin{array}{l}\text { Outcome } \\
\text { Measure }\end{array}$} & \multicolumn{4}{|c|}{ Task group } & \multicolumn{4}{|c|}{ Impairment group } & \multicolumn{2}{|c|}{ Between group comparison } \\
\hline & \multirow[t]{2}{*}{ Baseline } & \multirow{2}{*}{$\begin{array}{l}\text { Post-test } \\
\text { after } 4 \\
\text { weeks }\end{array}$} & \multicolumn{2}{|c|}{$\begin{array}{l}\text { Within-group } \\
\text { comparison }\end{array}$} & \multirow[t]{2}{*}{ Baseline } & \multirow{2}{*}{$\begin{array}{l}\text { Post-test } \\
\text { after } 4 \\
\text { weeks }\end{array}$} & \multicolumn{2}{|c|}{$\begin{array}{l}\text { Within-group } \\
\text { comparison }\end{array}$} & \multirow[t]{2}{*}{$p$ value } & \multirow{2}{*}{$\begin{array}{l}\text { Effect Size (Partial } \\
\text { Eta Squared) }\end{array}$} \\
\hline & & & Difference & $p$ value & & & Difference & $\overline{p \text { value }}$ & & \\
\hline $\operatorname{CGV}(\mathrm{m} / \mathrm{s})$ & $0.58 \pm 0.13$ & $0.69 \pm 0.11$ & $0.12 \pm 0.08$ & 0.003 & $0.77 \pm 0.31$ & $0.72 \pm 0.25$ & $-0.05 \pm 0.28$ & 0.61 & 0.29 & 0.072 \\
\hline $\mathrm{FGV}(\mathrm{m} / \mathrm{s})$ & $0.72 \pm 0.21$ & $0.98 \pm 0.33$ & $0.25 \pm 0.22$ & 0.007 & $0.94 \pm 0.40$ & $0.84 \pm 0.27$ & $-0.10 \pm 0.34$ & 0.38 & 0.07 & 0.207 \\
\hline TUDS (s) & $\begin{array}{c}65.21 \pm \\
45.47\end{array}$ & $\begin{array}{c}55.43 \pm \\
36.69\end{array}$ & $9.79 \pm 9.76$ & 0.35 & $\begin{array}{c}54.08 \pm \\
21.58\end{array}$ & $\begin{array}{c}51.18 \pm \\
21.57\end{array}$ & $2.90 \pm 1.81$ & 0.15 & 0.06 & 0.223 \\
\hline 6MWT (m) & $\begin{array}{c}196.40 \pm \\
70.70\end{array}$ & $\begin{array}{c}216.50 \pm \\
60.20\end{array}$ & $\begin{array}{c}20.10 \pm \\
27.60\end{array}$ & 0.06 & $\begin{array}{c}184.20 \pm \\
61.00\end{array}$ & $\begin{array}{l}210 \pm \\
70.40\end{array}$ & $25.80 \pm 31.2$ & 0.038 & 0.89 & 0.049 \\
\hline
\end{tabular}

* mean \pm standard deviation

** Alpha level was set at 0.05

*** CGV = comfortable gait velocity; FGV = fast gait velocity; TUDS = timed up and down stairs; 6MWT = six minute walk test

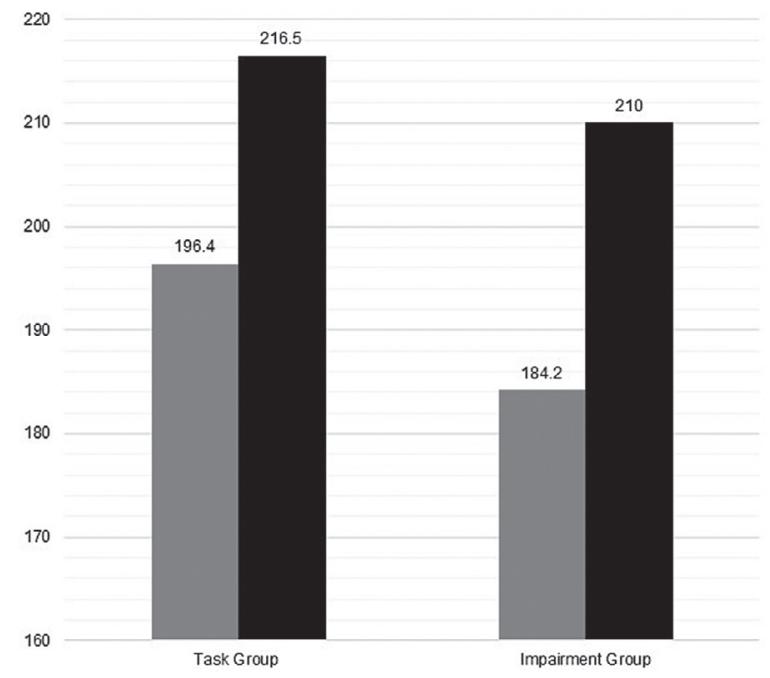

Legend: $\square$ Pretest $\mathbf{\square}$ Posttest

Figure 2. Comparison of the Six Minute Walk Test for both groups (meters).

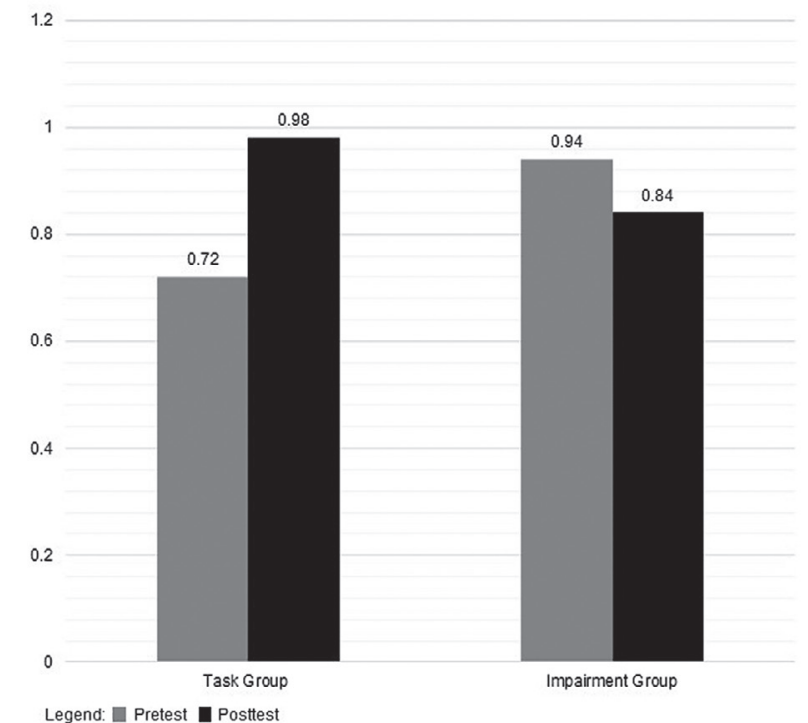

Figure 4. Comparison of the Fast Gait Velocity for both groups $(\mathrm{m} / \mathrm{s})$.

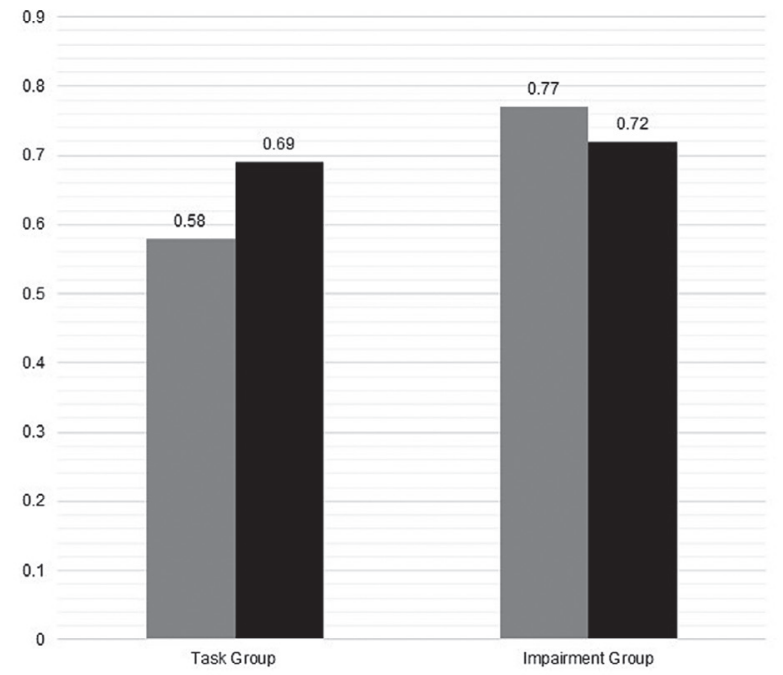

Legend: $\square$ Pretest $\square$ Posttest

Figure 3. Comparison of the Comfortable Gait Velocity for both groups $(\mathrm{m} / \mathrm{s})$.

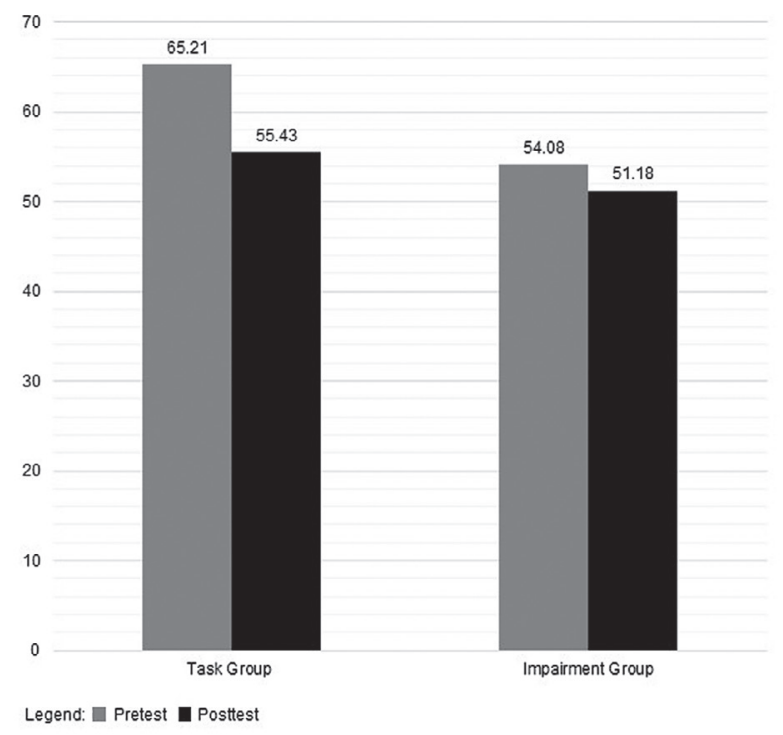

Figure 5. Comparison of the Timed Up and Down Stairs for both groups (seconds). 


\section{DISCUSSION}

This pilot study aimed to gather preliminary information to compare the effects of a task-oriented circuit class therapy and a circuit class therapy focused on specific impairments on the walking ability among patients with chronic stroke.

\section{Within-group change following circuit class therapy}

The effect of a circuit class therapy with stations focused on improving impairments on post-stroke walking ability has not been studied before. The results of this pilot study illustrate that this intervention shows promise in improving the ability to walk long distances. The intervention demonstrated statistically significant within-group changes for the 6MWT. Majority of the stations in this program focused on increasing muscle strength which might have consequently led to the increase in walking distance. Muscle leg strength of the affected extremity is considered as one of the important determinants in improving walking capacity among patients with chronic stroke. ${ }^{31}$ This finding demonstrated a potentially positive impact of impairmentfocused circuit class therapy on the overall function of patients because the 6MWT has shown to be a good predictor of community walking, and has shown significant correlation with quality of life after stroke. ${ }^{32,33}$

\section{Within-group change following task-oriented circuit class therapy}

Within group changes showed that the task-oriented circuit class therapy is effective in improving self-selected and fast gait velocity. Our findings confirmed what is already known regarding the benefits of this intervention. According to a systematic review by English et al in 2017, task-oriented circuit class therapy was more effective in improving gait velocity compared to other interventions and it yielded clinically significant results. ${ }^{15}$ This intervention allows for task specificity and high intensity of practice which are considered essential for improving walking. ${ }^{34,35}$ One of the stations in this program specifically targeted changing speed which provided the participants opportunities to practice this particular skill.

It is important to note, however, that the preliminary results also showed that the task-oriented circuit class therapy did not improve walking capacity. This intervention only has a $p$ value of 0.6 for the within-group change for the 6MWT. This is opposite of what is currently known in literature. Improvement in walking capacity is one of the consistent findings among studies about task-oriented circuit class training. ${ }^{15}$ The small sample size in the pilot study may have contributed to this result. Confirming this result would be better investigated in a future study with a larger sample size.

\section{Between-group change following both circuit class therapy}

Each of the circuit class therapy programs in this pilot study showed promising effects of specific aspects of walking ability, however, there was no significant difference between the effects of the two programs.

Task specificity and high intensity of practice are main factors that contribute to improved walking ability poststroke. $^{34,35}$ Although the function-based circuit programs have both of these elements, the impairment-focused circuit program also has the element of high intensity progressive training. According to literature, circuit class therapy provides high training intensity which is continually progressed in each succeeding station which may lead to improved limb function. ${ }^{10,11,12,35}$ Both programs in this pilot emphasized repetitive practice in each of the stations, regardless if the station is focused on functional tasks or exercises. This may have led to the same improvements seen in both programs. It may be worth exploring to see if circuit training as the delivery of intervention rather than the content itself of the circuit makes this model of intervention potentially effective.

Treatment focused on impairments improve the patient's bodily capacity to perform functional tasks. On the other hand, treatment focused on functional tasks improve the patient's capability in performing these tasks. Both types of intervention may contribute to better functional ability. ${ }^{17}$ In a study comparing individualized task-specific program and individualized impairment-focused treatment, the results revealed that both programs have similar effects in improving mobility post stroke. ${ }^{22}$ The result of the pilot study is consistent with this assertion. A recent systematic review also demonstrated that no approach is considered superior over the other in improving mobility after stroke. The review showed that physical therapy is more effective if the different approaches are mixed. ${ }^{21}$ However, this will need to be investigated formally if this pattern will still be observable in a proper well-designed clinical trial.

\section{Limitations and Recommendations}

The two circuit programs were shown to be feasible and safe to administer especially in a resource-limited setting. Both programs utilized readily available material seen in most physical therapy clinics. The protocol utilized at most two therapists per session provided that the number of patients undergoing a group session did not exceed five. It is possible for one therapist to safely monitor at most three patients undergoing the circuit at the same time. The need for resources is considerably less compared to one-on-one treatment. Moreover, there were no adverse reactions noted by the participants from any of the treatment programs.

The study has several limitations. Being a pilot study, the sample of patients was deliberately small and underpowered. Although, the small size expectedly affected the results of the study, we were still able to obtain some statistically significant findings. Our study used 4 weeks as the duration of the intervention which according to Jeon and colleagues, is enough to reveal medium effect sizes. ${ }^{5} \mathrm{We}$, however, still recommend using a longer training duration in future studies to determine if there would be any difference in the results. 
In terms of assessment, the present study only focused on functional measurements as patients with stroke perceive these as important indicators of recovery. ${ }^{36}$ However, since the impairments were not monitored, we might have missed some improvements in these areas brought about by the training. Moreover, we also recommend monitoring biomechanics and gait parameters using a more objective instrumented gait and motion analysis system. We also recommend conducting a follow-up assessment after the end of the program to see if any of the improvements will be maintained.

Although these limitations affect the generalizability of the results, we were able to gather information that increased our understanding of the circuit class therapy and the two approaches in stroke rehabilitation. Given the limits of the pilot study, we were able to test this research methodology which we found useful in conducting a future study with an appropriate sample size. ${ }^{37}$

\section{CONCLUSION}

Based on this pilot study, both circuit class therapy programs aimed at improving impairments and aimed at improving walking activities have promising effects on specific aspects of walking ability. Although, there was no significant difference between the two, our preliminary findings suggest that either a task-oriented circuit class therapy or an impairment-focused circuit class therapy may be useful. However, this will need to be confirmed using an appropriately-powered trial.

\section{Acknowledgments}

The authors acknowledge the assistance of Sophia Gaveria and Janine Escuadro in providing the interventions to the patients, and Alma Labro in assisting with the project management.

\section{Statement of Authorship}

All authors participated in data collection and analysis, and approved the final version submitted.

\section{Author Disclosure}

All authors declared no conflicts of interest.

\section{Funding Source}

This paper was self-funded by the authors and had no external funding.

\section{REFERENCES}

1. Feigin VL, Norrving B, Mensah GA. Global burden of stroke. Circ Res.2017 Feb; 120(3):439-48. doi: 10.1161/CIRCRESAHA. 116.308413 .

2. GBD 2016 Neurology Collaborators. Global, regional, and national burden of neurological disorders, 1990-2016: a systematic analysis for the Global Burden of Disease Study 2016. Lancet Neurol. 2019 May; 18(5)459-80. doi: 10.1016/S1474-4422(18)30499-X.
3. Poungvarin N. Stroke in the developing world. Lancet. 1998 Oct; 352 Suppl 3:SIII19-22. doi: 10.1016/s0140-6736(98)90090-3.

4. Nordin NAM, Aziz NAA, Aziz AFA, Singh DKA, Othman NAO, Sulong S, et al. Exploring views on long term rehabilitation for people with stroke in a developing country: findings from focus group discussions. BMC Health Serv Res. 2014 Mar; 14(1):118. doi: 10.1186/1472-6963-14-118.

5. World Confederation for Physical Therapy, Philippines: a profile of the profession [Internet]. 2018 [cited 2019 Apr]. Available from https://www.wcpt.org/node/150143/cds

6. Jeon BJ, Kim WH, Park EY. Effect of task-oriented training for people with stroke: a meta-analysis focused on repetitive or circuit training. Top Stroke Rehabil. 2015 Feb;22(1):34-43. doi: 10.1179/ 1074935714Z.0000000035.

7. Duncan PW, Zorowitz R, Bates B, Choi JY, Glasberg JJ, Graham GD, et al. Management of adult stroke rehabilitation care: a clinical practice guideline. Stroke. 2005 Sep; 36(9):e100-43. doi: 10.1161/01. STR.0000180861.54180.FF.

8. Perry J, Garrett M, Gronley JK, Mulroy SJ. Classification of walking handicap in the stroke population. Stroke. 1995 Jun; 26(6):982-9. doi: 10.1161/01.str.26.6.982.

9. Tyson SF, Hanley M, Chillala J, Selley A, Tallis RC. Balance disability after stroke. Phys Ther. 2006 Jan; 86(1):30-8. doi: 10.1093/ptj/86.1.30.

10. English CK, Hillier SL, Stiller KR, Warden-Flood A. Circuit class therapy versus individual physiotherapy sessions during inpatient stroke rehabilitation: a controlled trial. Arch Phys Med Rehabil. 2007 Aug; 88(8):955-63. doi: 10.1016/j.apmr.2007.04.010.

11. van de Port IGL, Wevers LEG, Lindeman E, Kwakkel G. Effects of circuit training as alternative to usual physiotherapy after stroke: randomised controlled trial. BMJ. 2012 May; 344:e2672. doi: 10.1136/bmj.e2672.

12. Wevers L, van de Port I, Vermue M, Mead G, Kwakkel G. Effects of task-oriented circuit class training on walking competency after stroke: a systematic review. Stroke. 2009 Jul;40(7):2450-9. doi: 10.1161/STROKEAHA.108.541946.

13. Dean CM, Richards CL, Malouin F. Task-related circuit training improves performance of locomotor tasks in chronic stroke: a randomized, controlled pilot trial. Arch Phys Med Rehabil. 2000 Apr; 81(4):409-17. doi: 10.1053/mr.2000.3839.

14. Veerbeek JM, van Wegen E, van Peppen R, van der Wees PJ, Hendriks E, Rietberg M, et al. What is the evidence for physical therapy poststroke? a systematic review and meta-analysis. PLoS One. 2014 Feb; 9(2):e87987. doi: 10.1371/journal.pone.0087987.

15. English C, Hillier SL, Lynch EA. Circuit class therapy for improving mobility after stroke. Cochrane Database Syst Rev. 2017 Jun; 6(6):CD007513. doi: 10.1002/14651858.CD007513.pub3.

16. Pang MYC, EngJJ, Dawson AS, McKay HA, Harris JE. A communitybased fitness and mobility exercise program for older adults with chronic stroke: a randomized, controlled trial. J Am Geriatr Soc. 2005 Oct; 53(10):1667-74. doi: 10.1111/j.1532-5415.2005.53521.x.

17. Sullivan KJ, Cen SY. Model of disablement and recovery: knowledge translation in rehabilitation research and practice. Phys Ther. 2011 Dec; 91(12):1892-904. doi: 10.2522/ptj.20110003.

18. Platz T. Impairment-oriented training (IOT) -- scientific concept and evidence-based treatment strategies. Restor Neurol Neurosci. 2004; 22(3-5):301-15.

19. Krebs HI, Mernoff S, Fasoli SE, Hughes R, Stein J, Hogan N. A comparison of functional and impairment-based robotic training in severe to moderate chronic stroke: a pilot study. NeuroRehabilitation. 2008; 23(1):81-7.

20. Platz T, Van Kaick S, Möller L, Freund S, Winter T, Kim IH. Impairment-oriented training and adaptive motor cortex reorganisation after stroke: a fTMS study. J Neurol. 2005 Nov;252(11): 1363-71. doi: 10.1007/s00415-005-0868-y.

21. Pollock A, Baer G, Campbell P, Choo PL, Forster A, Morris J, et al. Physical rehabilitation approaches for the recovery of function and mobility following stroke. Cochrane Database Syst Rev. 2014 Apr; 2014(4):CD001920. doi: 10.1002/14651858.CD001920.pub3. 
22. Duncan PW, Sullivan KJ, Behrman AL, Azen SP, Wu SS, Nadeau SE, et al. Body-weight-supported treadmill rehabilitation after stroke. N Engl J Med. 2011 May; 364(21):2026-36. doi: 10.1056/ NEJMoa1010790.

23. Portney LG, Watkins MP. Foundations of clinical research: applications to practice, 3rd ed. New Jersey: Pearson Prentice Hall; 2009. pp. 196199.

24. Shumway-Cook A, Patla AE, Stewart A, Ferrucci L, Ciol MA, Guralnik JM. Environmental demands associated with community mobility in older adults with and without mobility disabilities. Phys Ther. 2002 Jul; 82(7):670-81.

25. Eng JJ, Dawson AS, Chu KS. Submaximal exercise in persons with stroke: Test-retest reliability and concurrent validity with maximal oxygen consumption. Arch Phys Med Rehabil. 2004 Jan; 85(1):113-8. doi: 10.1016/s0003-9993(03)00436-2.

26. Flansbjer UB, Holmbäck AM, Downham D, Patten C, Lexell J. Reliability of gait performance tests in men and women with hemiparesis after stroke. J Rehabil Med. 2005 Mar; 37(2):75-82. doi: 10.1080/16501970410017215

27. Wolf SL, Catlin PA, Gage K, Gurucharri K, Robertson R, Stephen K. Establishing the reliability and validity of measurements of walking time using the Emory Functional Ambulation Profile. Phys Ther. 1999 Dec; 79(12):1122-33. doi:10.1093/ptj/79.12.1122.

28. Kosak M, Smith T. Comparison of the 2-, 6-, and 12-minute walk tests in patients with stroke. J Rehabil Res Dev. 2005 Jan-Feb; 42(1):103-7. doi: 10.1682/jrrd.2003.11.0171.

29. Hiengkaew V, Jitaree K, Chaiyawat P. Minimal detectable changes of the Berg Balance Scale, Fugl-Meyer Assessment Scale, Timed "Up \& Go" Test, gait speeds, and 2-minute walk test in individuals with chronic stroke with different degrees of ankle plantarflexor tone. Arch Phys Med Rehabil. 2012 Jul; 93(7):1201-8. doi: 10.1016/ j.apmr.2012.01.014.
30. Seguin R, Epping J, Bloch R, Buchner D, Nelson M. Growing stronger: strength training for older adults. Boston, MA: Tufts University; 2002. pp. 32-69.

31. Pang MYC, Eng JJ. Determinants of improvement in walking capacity among individuals with chronic stroke following a multi-dimensional exercise program. J Rehabil Med. 2008 Apr; 40(4):284-90. doi: 10.2340/16501977-0166.

32. Fulk GD, Reynolds C, Mondal S, Deutsch JE. Predicting home and community walking activity in people with stroke. Arch Phys Med Rehabil. 2010 Oct; 91(10):1582-6. doi: 10.1016/j.apmr.2010.07.005.

33. Muren MA, Hütler M, Hooper J. Functional capacity and healthrelated quality of life in individuals post stroke. Top Stroke Rehabil. 2008 Jan-Feb; 15(1):51-8. doi: 10.1310/tsr1501-51.

34. Kwakkel G. Impact of intensity of practice after stroke: issues for consideration. Disabil Rehabil. 2006 Jul; 28(13-14):823-30. doi: 10.1080/09638280500534861.

35. Kwakkel G, van Peppen R, Wagenaar RC, Dauphinee SW, Richards C, Ashburn A, et al. Effects of augmented exercise therapy time after stroke: a meta-analysis. Stroke. 2004 Nov; 35(11):2529-39. doi: 10.1161/01.STR.0000143153.76460.7d.

36. Doolittle ND. Stroke recovery: Review of the literature and suggestions for future research. J Neurosci Nurs. 1988 Jun; 20(3):169-73. doi: 10.1097/01376517-198806000-00007.

37. Leon AC, Davis LL, Kraemer HC. The role and interpretation of pilot studies in clinical research. J Psychiatr Res. 2011 May; 45(5): 626-9. doi: 10.1016/j.jpsychires.2010.10.008.

The Acta Medica Philippina is now accepting original scientific papers, review articles and case reports for its upcoming issues. Please follow the format for submission as indicated in the "Instructions to Authors" elsewhere in the journal. All papers received shall be properly acknowledged. For inquiries and submission of proposals, please email us at actamedicaphilippina.upm@up.edu.ph 